Department on Anaesthesia and Intensive Care and ${ }^{1}$ Neurosurgery, Postgraduate Institute of Medical Education and Research, Chandigarh, India

Background: To compare the efficacy of equimolar, equivolemic solutions of 3\% hypertonic saline and $20 \%$ mannitol on intraoperative brain relaxation in patients with clinical and radiological evidence of raised ICP undergoing surgery for supratentorial tumors. Materials and Methods: This study was carried out on 30 ASA I-III patients with age group ranging between 18-65 years, undergoing supratentorial tumor surgery. Patients received equimolar, equivolemic solutions of $3 \%$ hypertonic saline (osmolarity-1024) and 20\% mannitol (osmolarity-1098). Both the agents were administered at the dose of $5 \mathrm{ml} / \mathrm{kg}$ over a period of 15 minutes. Patients with previous history of electrolyte imbalance and getting hypertonic saline (HTS) prior to the surgery were excluded from the study. Brain relaxation was assessed by anesthesiologist (on a 3 point scale) and surgeon (on a 4 point scale). Results: Equimolar solutions of both mannitol and 3\% saline produced similar relaxation as assessed by surgeon and anesthesiologist. Urine output was more in mannitol group where as hypertonic saline group had increased serum sodium concentration, which returned to normal in 48 hours. CVP and mean arterial pressure were maintained close to the baseline in HTS group but CVP was higher in mannitol group but returned to normal in 3-4 hours. Conclusions: Since both these agents' mannitol and HTS have nearly equimolar concentration, they produce similar brain relaxation. Gemma et a., $l$ (1997) and Rozet et al., (2007) also had similar observations. Equimolar concentration of HTS and mannitol produce similar brain relaxation in patients undergoing surgery for supratentorial tumors.

\section{Effect of dexmedetomidine on postoperative recovery in patients undergoing cervical spine surgery}

\author{
Varun Jain, Arvind Chaturvedi, Mihir P Pandia, \\ Parmod K. Bithal \\ Department of Neuroanaesthesiology, AIIMS, New Delhi, \\ India
}

Background: The present study is planned to evaluate the effect of dexmedetomidine as an intraoperative anaesthetic adjuvant and its effect on postoperative extubation and recovery profile in patients undergoing anterior cervical spine surgeries. Primary objective was to assess postoperative recovery profile. Secondary objectives were to assess postoperative pain, time for need of first analgesic and to observe the perioperative hemodynamics. Materials and Methods: Thirty ASA I-II Adult patients (age 18-60 yrs) were randomized in this placebo controlled, double blind study. In the Dexmed Group, Dexmedetomidine was started at $0.2 \mu \mathrm{g} / \mathrm{kg} / \mathrm{hr}$ after a loading dose of $1 \mu \mathrm{g} / \mathrm{kg}$ before induction. Perioperative hemodynamics, intraoperative fentanyl and sevoflurane consumption, and postoperative recovery profile were observed by blinded observer. Postoperative pain and discharge readiness from post anesthesia care unit was evaluated using VRS score and modified Aldrete score, respectively. Results: Seventeen patients in placebo and 18 in dexmedetomidine group were enrolled. Time to emergence, extubation and to achieve modified Alderte score $\geq 9$ was earlier in Dexmed group (mean 7.8 minutes; 9.8 minutes; 4.5 minutes) compared to Placebo group (10.5 minutes; 13.2 minutes; 13.7 minutes) $(P=0.01)$. Pain score at extubation was lower (2.8 vs. 26.2) and time for first ${ }^{t}$ analgesic longer (46.6 minutes vs. 18.7 minutes) in Dexmed group compared to placebo. Hemodynamics was better controlled with Dexmedetomidine. Conclusion: Use of Dexmedetomidine in such low dose for abolishing cough and pressor response has not been described previously in cervical spine surgeries where smooth emergence and extubation is desirable. Intraoperative use of dexmedetomidine at lowest recommended dosage in adults undergoing anterior cervical spine surgery results in a favorable recovery profile with reduced emergence/ extubation time and postoperative pain, without adverse perioperative hemodynamic effects.

\section{To evaluate the effects of dexmedetomidine on intraocular pressure and hemodynamic changes in response to laryngoscopy and tracheal intubation and its influences on anesthetic requirements during intracranial tumor surgery}

Tanuja Trivedi, Shobha Purohit

Department of Anaesthesia, SMS Medical College, Jaipur, Maharashtra, India

Background: Brain relaxation is one of the most important prerequisites for neurosurgeries. The other points of concern are the need of stable hemodynamics with less fluctuation in ICP and speedy recovery from anesthesia. Endotracheal intubation is one of the major stressful stimuli inside an operation theatre that can elicit a marked pressor response. Various drugs have been used to attenuate these reflexes and reduce hemodynamic changes. $\alpha_{2}$-Agonist are a novel class of drugs They have neuroprotective, cardioprotective, and sedative effects. These unique characteristics make them potentially useful during neuroanesthesia. Recent studies have shown that Dexmedetomidine is able to decrease circulating plasma norepinephrine and epinephrine concentration 
in approximately $50 \%$, decreases brain blood flow by directly acting on post-synaptic $\alpha 2$ receptors, decreases CSF pressure without ischemic suffering and effectively decreases brain metabolism and intracranial pressure and also able to decrease injury caused by focal ischemia. Materials and Methods: A prospective randomized study was done on 50 patients (ASA grade 1, 2) scheduled for intracranial tumor surgeries were divided into study or control group (25 each). Both group received Midazolam $(0.02 \mathrm{mg} / \mathrm{kg})$ iv, Glycopyrrolate $(0.005 \mathrm{mg} / \mathrm{kg})$ iv, Inj Fentanyl $(2 \mu \mathrm{g} / \mathrm{kg})$ iv and group D received dexmedetomidine $0.8 \mu \mathrm{g} / \mathrm{kg}$ iv in $20 \mathrm{ml}$ saline over 10 mins and group $C$ received $20 \mathrm{ml}$ Normal saline iv for same time period without dexmedetomidine in Premedication. Patients were induced with inj. Propofol (iv) after giving test drug and dose was calculated by BIS values between 40-60, followed by inj. Vecuronium $0.1 \mathrm{mg} / \mathrm{kg}$ after recording hemodynamic measurements. Patients were ventilated with 100\% oxygen for 180 seconds. Hemodynamic measurements were recorded two minutes before test drug and placebo, two minutes before and one minutes after induction and just after and one minutes, two minutes, five minutes and 10 minutes of intubation. Change in intraocular pressure with the help of schiotz tonometer is also noted during this period. The groups were compared with heart rate, systolic blood pressure, and diastolic blood pressure and mean arterial pressure, IOP and IV dose of Propofol requirement for induction. Results: Groups were well matched for their demographic data $(P>0.05)$ and preoperative vitals. Heart rate and mean arterial blood pressure decreased significantly in patients of group D (Dexmedetomidine group) compared to group $C$ (placebo group) $(P<0.05)$, and patients were more hemodynamicaly stable before induction, and at T-1, T-2, T-5, T-10 minutes after induction in group $\mathrm{D}(P$ value $<0.05)$. The total propofol requirements for induction is more in patients of group $C$ than in patients of group D $(128.6 \pm 15.5 \mathrm{mg}$ vs. $91.8 \pm 20.41 \mathrm{mg}$, $P<0.05)$. The intraocular pressure in right eye and left eye decreases significantly from pre-induction value of 21.97 $\pm 4.75 \mathrm{~mm} \mathrm{Hg}$ and $22.08 \pm 4.94 \mathrm{~mm} \mathrm{Hg}$ to $18.02 \pm 4.30$ $\mathrm{mm} \mathrm{Hg}$ at intubation and $17.78 \pm 4.15 \mathrm{~mm} \mathrm{Hg}$ one minutes after intubation in both the eyes in group $\mathrm{D}$ after the test drug. In Group C IOP at pre-induction, intubation and one minute after intubation are $20.96 \pm 3.80 \mathrm{~mm} \mathrm{Hg}, 26.55$ $\pm 4.08 \mathrm{~mm} \mathrm{Hg}$ and $25.99 \pm 3.78 \mathrm{~mm} \mathrm{Hg}$ in right eye and $21.05 \pm 3.72 \mathrm{~mm} \mathrm{Hg}, 26.38 \pm 4.14$ and $25.90 \pm 3.75 \mathrm{~mm} \mathrm{Hg}$ in left eye respectively and the difference is statistically significant between the groups. Bradycardia and hypotension incidences were higher in group D. Conclusion: Dexmedetomidine is an excellent drug when used as an adjunct to general anesthesia for attenuation of pressor response. It not only decreased the magnitude of stress response to intubation but also decreased the intraocular pressure and induction dose requirement of propofol.

\section{Role of Intravenous Magnesium sulphate in spinal surgery for hypotensive anesthesia}

\author{
M . Somani, S. Sachdev, V. Mathur, D. D. Jethava, \\ D. Jethava
}

Department of Anaesthesiology and Critical Care, Mahatma Gandhi Medical College, Jaipur, Rajasthan, India

Background: This randomized, double-blind placebo controlled study was undertaken to evaluate effect of intravenous magnesium sulphate for hypotensive anesthesia in patient for spinal surgery. Materials and Methods: Sixty patients undergoing spinal surgery were included in two parallel groups. The magnesium group $(\mathrm{M})$ received magnesium sulphate $40 \mathrm{mg} / \mathrm{kg}$ as a bolus before induction of anesthesia and $10 \mathrm{mg} / \mathrm{kg} / \mathrm{hr}$ by continuous infusion during operation through out surgery. The control group (C) as above received isotonic saline solution both as bolus and infusion at the same rate. Result: The surgical time was reduced in magnesium group [170 (40) minutes] vs. [180 (45) minutes] in control group. The anesthetic requirements (fentanyl, vecuronium, isoflurane), mean arterial pressure $(P<0.005)$ and heart rate $(P<0.005)$ were also significantly reduced. However, the anesthetic time was longer as observed thus a prolongation in emergence from anaesthesia. Conclusion: Intravenous magnesium sulphate led to a significant decrease in mean arterial pressure, heart rate, and duration of surgery. It also alters anesthetic dose requirements.

\section{Intraoperative management of cerebral arteriovenous malformations: Our experience}

\author{
Harshal Dholke, Mohan Rao, Manas Panigrahi \\ Department of Anaesthesia and ${ }^{1}$ Neurosurgery, \\ Krishna Institute of Medical Sciences. Secundrabad, \\ Andhra Pradesh, India
}

Background: To discuss the institutional experience in the intraoperative management of symptomatic patients of cerebral AVM surgery. Materials and Methods: It is a retrospective descriptive study done in patients with cerebral AVMs who were managed by surgery at KIMS hospital between Janaury 2010 to July 2013. Totally 20 patients are included in this study. Results: Of the total patients who underwent surgery there were equal no of patients who had bleeding and who did not. Of the 